JPPT I Critical Care

\title{
Prolonged Dexmedetomidine Infusion and Drug Withdrawal In Critically Ill Children
}

Astrid S. Haenecour, MD; Winnie Seto, MSc; Charline M. Urbain, PhD; Derek Stephens, MSc; Peter C. Laussen, MD; and Corrine R. Balit, MD

OBJECTIVE To characterise the incidence, symptoms and risk factors for withdrawal associated with prolonged dexmedetomidine infusion in paediatric critically ill patients.

METHODS Retrospective chart review in the paediatric intensive care unit and the cardiac critical care unit of a single tertiary children's hospital. Patients up to 18 years old, who received dexmedetomidine for longer than 48 hours were included.

RESULTS A total of 52 patients accounted for 68 unique dexmedetomidine treatment courses of more than 48 hours. We identified 24 separate episodes of withdrawal in the 68 dexmedetomidine courses (incidence $35 \%)$. Of these episodes $38 \%$ occurred in patients who were weaned from dexmedetomidine alone while the remaining occurred in patients who had concurrent weans of opioids and/or benzodiazepines. Most common symptoms were agitation, fever, vomiting/retching, loose stools and decreased sleep. The symptoms occurred during the latter part of the wean or after discontinuation of dexmedetomidine. A cumulative dose of dexmedetomidine of $107 \mathrm{mcg} / \mathrm{kg}$ prior to initiation of wean was more likely associated with withdrawal (this equates to a dexmedetomidine infusion running at $1 \mathrm{mcg} / \mathrm{kg} / \mathrm{hr}$ over 4 days). Duration of opioid use was an additional risk factor for withdrawal. The use of clonidine, as a transition from dexmedetomidine, did not protect against withdrawal $(p=1)$.

CONCLUSIONS A withdrawal syndrome may occur after prolonged infusion of dexmedetomidine. As all our patients were also exposed to opioids this may be affected by the duration of opioid use. We identified a cumulative dose of 107 micrograms/kg of dexmedetomidine beyond which withdrawal symptoms were more likely (which equates to 4 days of use at a dose of $1 \mathrm{mcg} / \mathrm{kg} / \mathrm{hr}$ ). A protocol for weaning should be considered in this circumstance.

ABBREVIATIONS CCU, Cardiac Critical Care Unit; PICU, Pediatric Intensive Care Unit; ROC, Receiver operating characteristic; WAT-1, Withdrawal Assessment Tool-1

KEYWORDS dexmedetomidine; infusion; intensive care unit; intravenous; pediatric; substance withdrawal syndrome

J Pediatr Pharmacol Ther 2017;22(6):453-460

DOI: 10.5863/1551-6776-22.6.453

\section{Introduction}

Analgesia and sedation of paediatric critical care patients is essential to treat pain, reduce stress response and ensure patient safety. However, challenges to achieve this goal are well described with complications such as over sedation, delirium and drug withdrawal. There has been increasing use of dexmedetomidine in pediatric critical care to achieve this balance of benefit and minimise complications. ${ }^{2-4}$

Dexmedetomidine is a peripheral and central selective alpha- 2 adrenergic receptor agonist that has rapid onset and offset of action. ${ }^{5}$ Dexmedetomidine has anxiolytic and sedative effect mediated by its activity in the central nervous system (locus ceruleus) and analgesic effect from its activity on the peripheral nervous system (dorsal horn of the spinal cord). It induces a sedation promoting sleep pathway but with preserved respiratory drive ${ }^{3}$.

Dexmedetomidine was intended for short-term use with approval initially for 24 hours use. ${ }^{6}$ However, in clinical practice dexmedetomidine is used for longer periods and there are multiple reports describing this in paediatric critical care. ${ }^{7-10}$ Prolonged use of any sedative raises the question of drug withdrawal, which is well described with traditional analgesic and sedatives. ${ }^{1}$ This association with prolonged dexmedetomidine exposure is unclear. A recent retrospective study described an incidence of withdrawal of $30 \%$ after prolonged infusion of dexmedetomidine in children ${ }^{10}$ and this is supported by several case reports..$^{4,712-15}$ Equally there are some reports in the literature that do not describe withdrawal syndrome even with abrupt discontinuation of prolonged dexmedetomidine infusions. $8,9,16,17$ Symptoms frequently reported in the withdrawal from dexmedetomidine include anxiety, agitation, decreased 
sleep, loose stools, emesis, tremors and increased secretions. ${ }^{4,710}$ Risk factors for withdrawal are not known and there is no clear evidence for the best way to wean dexmedetomidine. Clonidine, an oral alpha- 2 agonist, may have a role in preventing or treating withdrawal from dexmedetomidine. There are some recommendations for the use of clonidine while slowly tapering dexmedetomidine infusions but there is no evidence about its effect on dexmedetomidine withdrawal. 18

The objective of our study was to characterise the incidence, symptoms and risk factors for withdrawal associated with prolonged dexmedetomidine exposure in critically ill children.

\section{Materials and Method}

This was a retrospective study of prolonged dexmedetomidine infusion in the Paediatric Intensive Care Unit (PICU) and Cardiac Critical Care Unit (CCCU) of a single tertiary children's hospital, between January $1^{\text {st }}$ and July $15^{\text {th }} 2014$.

Dexmedetomidine treatment courses were identified from the pharmacy database (Centricity BDM system, GE Healthcare, Chicago, Ilinois, United states of America) and then cross-referenced against the critical care database (Clinical Information Management System, Eclipsys of Atlanta, Georgia, Unites States of America) to verify the administration details. Courses taking place in patients aged less than 18 years old and for duration longer than 48 hours were included. Treatment courses were excluded if the patient received muscle relaxation during the 24 hours after discontinuation of dexmedetomidine infusion. Patients were also excluded if they were discharged from the unit or died during the period of dexmedetomidine use or in the 24 hours period following discontinuation of dexmedetomidine, as in these situations it would not be possible to assess for withdrawal symptoms. We included multiple dexmedetomidine courses in the same patient and treated these as separate treatment courses for analysis if the treatment courses were independent in time (at least 24 hours between discontinuation and restart) and if dexmedetomidine was restarted for sedation related to a clinical change and not for withdrawal issues.

PICU and CCCU patients receive opioids as first line analgesia with escalation to other agents for analgesia and sedation including dexmedetomidine at the discretion of the responsible physician. Dosage recommendation for dexmedetomidine was a loading dose of $0.5 \mathrm{mcg} / \mathrm{kg}$ with an infusion start dose of $0.5 \mathrm{mcg} /$ $\mathrm{kg} / \mathrm{hr}$. Infusion was increased to effect with a maximum recommended dose of $2 \mathrm{mcg} / \mathrm{kg} / \mathrm{hr}$. While the unit has an established algorithm for weaning of opioids and benzodiazepines, there was no standardized plan for discontinuation of dexmedetomidine.

Data collected included patient demographics (age, sex, weight), admission diagnosis, co-morbidities, dexmedetomidine details (bolus dose, infusion dose, cumulative dose, duration, previous exposure to dexmedetomidine) and other analgesic and sedative use prior, during and after the wean of dexmedetomidine. Detailed information was collected on the weaning period of dexmedetomidine (from 24 hours prior to wean to 24 hours after ceasing the infusion) and included: type of discontinuation (abrupt or weaned), duration of wean, doses at each step of wean, weaning interruption and reason for weaning interruption. The weaning start dose and time was determined by a documented decrease in the infusion rate and correlated in the nursing or medical notes as being completed with the intention to wean sedation. We defined the discontinuation of the infusion as abrupt if the infusion was stopped within one hour.

Assessment for potential episodes of withdrawal occurred during the weaning period and for 24 hours after cessation of the dexmedetomidine infusion. This time frame was based on the short elimination half-life of dexmedetomidine and the timing of withdrawal onset reported in the literature. ${ }^{12,14}$ Two methods were used to identify potential withdrawal episodes: reviewing nursing and medical notes and reviewing Withdrawal Assessment Tool-1 (WAT-1) scores. For the review of notes symptoms and signs extracted included agitated or unsettled behaviour, decreased sleep, emesis, diarrhoea, diaphoresis, fever, tremors and seizure or any new neurological symptom. Administration and indication of any additional analgesics and sedatives during the weaning period was also collected as well as any reference to discontinuation or hold of the weaning plan and reasons for this.

WAT-1 score is a validated withdrawal score for critically ill children over 1 month of age weaning from opioids and benzodiazepines. ${ }^{19}$ It is a 12-point score made up of 11 individual components that look at common withdrawal symptoms. When WAT-1 scores were available the total score as well as all the individual components were collected. This was done as an additional screening tool to ensure withdrawal symptoms were not missed.

All potential withdrawal episodes were reviewed by two investigators ( $\mathrm{AH}$ and $\mathrm{CB}$ ) to ensure that the symptoms reported were due to withdrawal and not to a clinical change in the patient. They screened for sepsis, change in cardiac status, mechanical ventilation changes, feed intolerance or some new pathology that could explain the development of these symptoms. If no new clinical state was identified then the symptoms were attributed to the wean of the medications. Hence, episodes where classified into the following categories: No withdrawal (the symptoms were related to underlying clinical state) or Withdrawal. Both reviewers had to agree on the diagnosis of withdrawal. Withdrawal episodes were then characterized as related either to dexmedetomidine alone (if that was the only drug that was weaned) or to dexmedetomidine plus other anal- 
gesic and sedatives if concurrent weans had occurred. Data from these different groups were compared.

We also extracted information on the use of clonidine that was started prophylactically at the beginning of the weaning period. We recorded the daily dose given and the overlap period between clonidine and dexmedetomidine.

Primary outcome was the presence or absence of withdrawal after prolonged dexmedetomidine infusion and the clinical symptoms reported. Secondary outcomes included risk factors that contributed to withdrawal and the role of clonidine in prevention of withdrawal.

Data were entered using excel 2011 (Microsoft, Redmond, Washington, United States of America). Statistics were completed using SAS Version 9.3 software (SAS Institute, Cary, North Caroline, United States of America). Categorical and continuous data were compared using Chi-square and t-tests as appropriate. If distributions were skewed then non-parametric tests were used. Satterthwaite adjustment was used when the variance were unequal. Results are expressed as mean with standard deviation or median with interquartile ranges (IQR). Univariate logistic regression was used to screen for possible risk factors, setting the $p$ value at 0.20 significance level for entry in the multiple regression model. Multiple logistic regression was then used to identify risk factors associated with withdrawal. A $p$ value of $<0.05$ was considered significant at the multiple regression stage. Receiver operating characteristic (ROC) analysis were performed using SAS and NCSS 2007 (NCSS Statistical Software, State College, Pennsylvania United States of America) to identify any significant cut-offs for withdrawal for cumulative dose of dexmedetomidine prior to weaning and duration of exposure to dexmedetomidine prior to weaning.

The study was approved by our institutional research ethics board. The need for informed consent was waived in view of the retrospective nature of the study.

\section{Results}

A total of 133 independent courses of dexmedetomidine were prescribed from January $1^{\text {st }} 2014$ to July $15^{\text {th }}$ 2014 , of which 68 courses fulfilled the criteria for enrolment in the study (Figure 1). These courses were prescribed in 52 patients with 9 patients having received multiple, but independent dexmedetomidine courses.

Characteristics of dexmedetomidine courses. Median age at the time of dexmedetomidine infusion was 5 months (range 1 day to 3 years), with a median weight of $5.1 \mathrm{~kg}$ (IQR 4.1-6.6). 54\% of the courses occurred in females. Main admission diagnosis was a primary cardiac disease (88.2\%) with $19.1 \%$ having single ventricle physiology and $7.4 \%$ presenting with cardiomyopathy. Twenty-seven (40\%) courses occurred in patients that had been exposed to dexmedetomidine over the previous year. Dexmedetomidine was used in the immediate
Figure 1. Flow diagram with inclusion and exclusion of dexmedetomidine treatment courses.

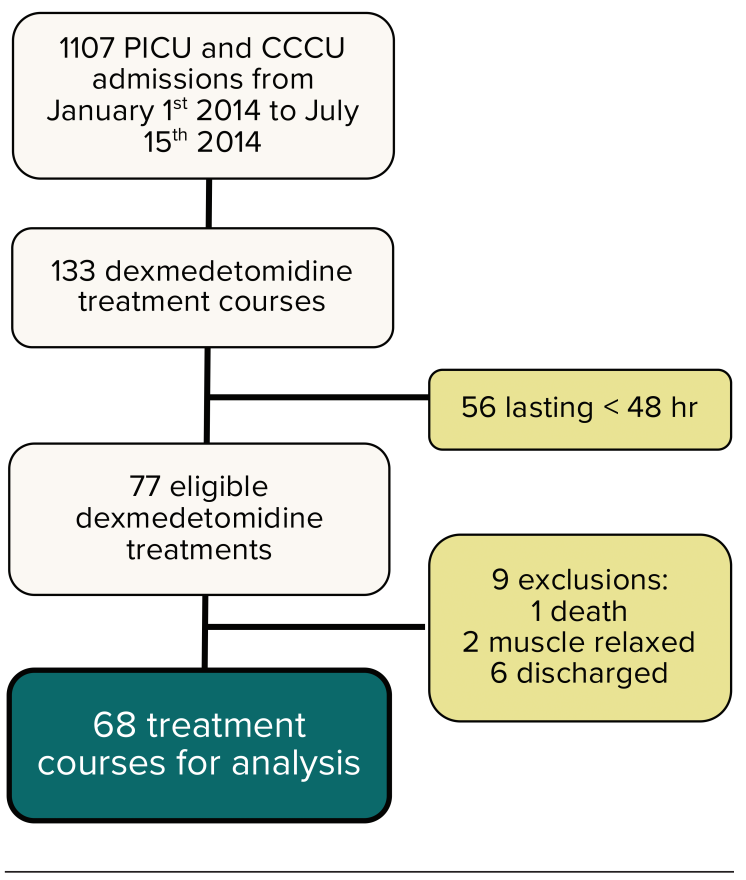

post-operative period in 55 (81\%) of the courses.

Dexmedetomidine exposure. Dexmedetomidine dose ranged from 0.1 to $2 \mathrm{mcg} / \mathrm{kg} / \mathrm{hr}$ with a median total cumulative dose of $86.7 \mathrm{mcg} / \mathrm{kg}$ (IQR 51.8-158.1). Median total duration of exposure to dexmedetomidine was 124.3 hours (IQR 75.9-178.5). The median duration of dexmedetomidine use prior to starting the wean was 96 hours (IQR 51.5-137), with a median cumulative dose prior to starting the wean of 72.6 microgram $/ \mathrm{kg}$ (IQR 41-131.2).

Concurrent use of other sedatives and their duration of use prior to the dexmedetomidine wean are reported in Table 1. There is no patient in our study that was exposed to dexmedetomidine alone.

Dexmedetomidine weaning. In 8 courses (11.8\%), dexmedetomidine discontinuation was abrupt. In the remaining cases, median duration of dexmedetomidine wean was 23 hours (IQR 14.1-42.1). Dexmedetomidine courses that were abruptly discontinued had a median duration of treatment prior to wean of 57.5 hours (IQR 48.6-84.2) and a median cumulative dose prior to wean of $63.5 \mathrm{mcg} / \mathrm{kg}$ (IQR 41.1-71.4). In the other 60 courses where dexmedetomidine was weaned, the median duration prior to starting the wean was 103 hours (IQR 68.5-157.1) with a mean cumulative dose of $89 \mathrm{mcg} / \mathrm{kg}$ (IQR 41-160.2) prior to weaning. In the majority of cases where weaning occurred it was by $0.2 \mathrm{mcg} / \mathrm{kg} / \mathrm{hr}$ every $6-8$ hours but was at the discretion of the treating team. The median duration of the wean was 24 hours (IQR 14.1-42.1). Dexmedetomidine was the only drug weaned or stopped in 26 of the courses (38.2\%). In the remain- 
Table 1. Concurrent Sedatives Use During Each Dexmedetomidine Treatment Course Prior to Weaning

\begin{tabular}{lcc} 
Sedatives & $\begin{array}{c}\text { Concurrent Sedative } \\
\mathbf{n}(\%)\end{array}$ & $\begin{array}{c}\text { Duration of Exposure (days) } \\
\text { Median (IQR) }\end{array}$ \\
Opioid & $68(100)$ & $9(5-17)$ \\
Benzodiazepine & $49(72)$ & $5(2.5-23.5)$ \\
Chloral Hydrate & $7(10.3)$ & $3(2-4)$ \\
\hline
\end{tabular}

$I Q R$, interquartile range

ing 42 courses (61.8\%) opioids and/or benzodiazepines were concurrently weaned with dexmedetomidine.

Clonidine was prescribed enterally to pre-empt possible withdrawal symptoms during the wean process in 37 treatment courses (54.4\%). The median total oral daily dose of clonidine used was $16 \mathrm{mcg} / \mathrm{kg}$ (IQR 12-20). The median overlap time of Clonidine and dexmedetomidine was 30 hours (IQR19.9-50.3).

Dexmedetomidine withdrawal. We found 49 potential episodes of withdrawal. After review, we excluded 25 episodes: 23 were explained by change in clinical status, one was related to opioid weaning alone and one was related to benzodiazepine wean alone. We then included 24 separate episodes of withdrawal, which represents an incidence of withdrawal of 35\% $(24 / 68)$. In the group that weaned from dexmedetomidine alone, the incidence of withdrawal was similar with 9 out of 26 courses developing withdrawal (35\%). In 11 courses (46\%) withdrawal appeared during the weaning period and in 13 courses it appeared after the infusion was discontinued. When withdrawal occurred during the weaning period, onset of symptoms was found after a median reduction in dexmedetomidine of $60 \%$ of the original dose (IQR 50-77) with a median time to onset of symptoms of 21 hours (IQR 7-24). When withdrawal occurred after the discontinuation of the infusion the median time to onset of symptoms was 11.5 hours (IQR 3.5-14.25).

Agitation and fever were the most commonly reported symptoms occurring $100 \%$ and $68 \%$ of the time, respectively. Symptoms were nearly similar in the group that only weaned from dexmedetomidine or who had concurrent weans (Table 2). Patients with courses who only weaned from dexmedetomidine had more decreased sleep then those that had a mixed wean. Median WAT-1 scores were 4 (IQR 3.25-6.5) in the group that just weaned from dexmedetomidine alone, compared to 6 (IQR 4-6) in the group with mixed weans.

Risk factors for withdrawal. The characteristics between courses where withdrawal developed and courses where there was no withdrawal are shown in Table 3. Factors associated with withdrawal included total cumulative dose of dexmedetomidine $(p=0.039)$ and cumulative dose of dexmedetomidine prior to starting the wean ( $p=0.059)$, duration of concurrent opioid treatment prior to the wean $(p=0.048)$, total duration of dexmedetomidine ( $p=0.037$ ) use as well as male sex $(p=0.003)$. The administration of clonidine did not

Table 2. Symptoms During the Weaning of Dexmedetomidine Alone $(N=9)$ and Mixed Weans $(n=15)$

\begin{tabular}{lcc} 
& DEX alone weans & Mixed weans* \\
Agitation & $\mathbf{n}(\%)$ & $\mathbf{n}(\%)$ \\
Fever & $9(100)$ & $15(100)$ \\
Vomiting/Wretching & $7(77.8)$ & $9(60)$ \\
Loose Stools/ Diarrhoea & $3(33.3)$ & $8(53.3)$ \\
Decreased Sleep & $4(44.4)$ & $3(20)$ \\
Tremors/jittery & $4(44.4)$ & $1(6.7)$ \\
Diaphroesis & $2(22.2)$ & $6(40)$ \\
Yawn/Sneeze & $3(33.3)$ & $5(33.3)$ \\
Uncoordinated /repeated movements & $0(0)$ & $2(13.3)$ \\
Startling to touch & $3(33.3)$ & $5(33.3)$ \\
Increased tone & $2(22.2)$ & $5(33.3)$ \\
Other ${ }^{+}$ & $3(33.3)$ & $3(20)$ \\
\hline
\end{tabular}

DEX, dexmedetomidine

* concurrent Administration of Opioids/Benzodiazepine

${ }^{+}$hiccups, episodes of staring 
Table 3. Comparison Between Groups Presenting With and Without Symptoms of Withdrawal From

Dexmedetomidine

\begin{tabular}{lccc} 
& $\begin{array}{c}\text { No Withdrawal } \\
\mathbf{n}=\mathbf{4 4}\end{array}$ & $\begin{array}{c}\text { Withdrawal } \\
\mathbf{n}=\mathbf{2 4}\end{array}$ & p value \\
\hline Age, rr $^{*}$ & $0.51 \pm 0.63$ & $0.61 \pm 0.59$ & 0.527 \\
Weight, kg* & $5.57 \pm 2.64$ & $6.05 \pm 2.46$ & 0.460 \\
Sex (Male) & 14 & 17 & $\mathbf{0 . 0 0 3}$ \\
Previous use of DEX, $\mathrm{n}$ & 17 & 10 & 1.000 \\
Abrupt discontinuation, $\mathrm{n}$ & 7 & 1 & 0.244 \\
Opioids duration prior to wean (days)* & $14.27 \pm 21.33$ & $\mathbf{0 . 0 4 8}$ \\
Prophylactic use of clonidine & 24 & 13 & 1.000 \\
DEX Dosing & & $193.81 \pm 194.37$ & $\mathbf{0 . 0 3 9}$ \\
Total cumulative dose, mcg/kg* & $103.43 \pm 88.39$ & $166.6 \pm 171.9$ & $\mathbf{0 . 0 5 9}$ \\
Cumulative dose prior to wean, $\mathrm{mcg} / \mathrm{kg}^{*}$ & $93.03 \pm 86.17$ & $187.86 \pm 120.59$ & $\mathbf{0 . 0 3 7}$ \\
Total duration (hrs)* & $127.74 \pm 86.26$ & $141.5 \pm 99.31$ & 0.154 \\
Duration prior to wean (hrs)* & $108.9 \pm 82.77$ & & \\
\hline
\end{tabular}

DEX, dexmedetomidine

* Mean \pm SD

show any effect on the occurrence of withdrawal $(p=1)$. In univariate and multiple logistic regression, the duration of opioid use prior to starting the wean, as well as cumulative dose of dexmedetomidine prior to starting the wean remained significant (Table 4). Other factors did not remain statistically significant.

A receiver operator curve $(\mathrm{ROC})$ analysis was performed for the duration of exposure to dexmedetomidine and the cumulative dose prior to starting to wean to examine whether it was possible to predict a cut off point for these variables beyond which withdrawal was more likely. A cumulative dose of $107 \mathrm{mcg} / \mathrm{kg}$ of dexmedetomidine prior to initiating the wean was associated with withdrawal with a sensitivity of $58 \%$ and a specificity of $77 \%$ (AUC $=0.65,95 \%$ Confidence interval 0.49-0.78) (Figure 2). ROC analysis of duration of dexmedetomidine prior to the wean did not show a cut off value.

\section{Discussion}

The use of dexmedetomidine is variable and in our experience takes place in complex patients who have not responded to usual first line analgesics and sedatives. In this retrospective analysis, we found withdrawal symptoms occurred in 35\% of prolonged dexmedetomidine courses. Findings in this study include confirmation of symptoms associated with dexmedetomidine withdrawal, and the possible identification of a cumulative dose of dexmedetomidine as a predictor of withdrawal.

Dexmedetomidine has rapid onset and offset of action with a terminal elimination half-life of approximately 2 hours. However, with prolonged infusion, dexmedetomidine has a context sensitive half-life to over 4 hours after an eight-hour infusion ${ }^{5}$. It has a large volume of distribution in children and is $94 \%$ protein bound ${ }^{5}$. Clearance of dexmedetomidine matures with age, reaching maturation at around 1 year and is also reduced by $27 \%$ after cardiac surgery. ${ }^{5}$ Based on these pharmacokinetics characteristics, we selected an observation period up to $24 \mathrm{~h}$ after discontinuation of the infusion to detect withdrawal symptoms. Our cohort of patients was predominately young (under 1 year) and mostly post cardiac surgery and hence we could expect decreased clearance of dexmedetomidine. This can explain why in our cohort withdrawal either occurred during the latter part of the infusion reduction (after a reduction of at least $60 \%$ of the starting dose) or after discontinuation of the infusion with a median time to onset of symptoms of 11.5 hours.

The most comparable case series by Whalen etal ${ }^{10}$ reported similar symptoms of withdrawal from dexmedetomidine with an incidence of $30 \%$. In our cohort, patients had much larger doses of dexmedetomidine and were much younger with median age in our study of 5 months compared to 3.8 years. Our median maximal dose was $1.2 \mathrm{mcg} / \mathrm{kg} / \mathrm{hr}$ (IQR 1-1.5) compared to 0.7 $\mathrm{mcg} / \mathrm{kg} / \mathrm{h}$ (IQR 0.5-1.5) in Whalen's series with a median duration of infusion in the same range (124 versus 141 hours). This may explain why we had a slightly higher incidence of withdrawal in our cohort.

Agitation, fever and emesis were the most reported symptoms with withdrawal. This is consistent with previous reports in the literature ${ }^{4,7,10,12-15}$ with agitation being reported in all cases. Fever occurred more frequently in our population. We did look at symptoms and WAT scores in the groups that only had dexmedetomidine weaned and those that had concurrent weans showing 


\begin{tabular}{|c|c|c|}
\hline & $\begin{array}{l}\text { Odds Ratio } \\
\text { (95\% Cl) }\end{array}$ & $\mathrm{p}$ value \\
\hline \multicolumn{3}{|l|}{ Univariate logistic regression } \\
\hline Cumulative DEX dose before wean $(\mathrm{mcg} / \mathrm{kg})$ & $1.005(1.001,1.010)$ & 0.039 \\
\hline Duration of DEX before wean (hrs) & $1.004(0.998,1.010)$ & 0.158 \\
\hline Opioids duration prior to wean (days) & $1.020(1.001-1.037)$ & 0.033 \\
\hline \multicolumn{3}{|l|}{ Multiple logistic regression } \\
\hline Cumulative DEX dose before wean (mcg/kg) & $1.005(1.001,1.010)$ & 0.032 \\
\hline Opioids duration prior to wean (days) & $1.020(1.002,1.038)$ & 0.028 \\
\hline
\end{tabular}

$C l$, Confidence interval; DEX, dexmedetomidine

that the clinical features are similar. Of particular interest, one symptom that seems to be more specific to dexmedetomidine is decreased sleep on discontinuation of the infusion. This occurred in $44 \%$ of our patients and has previously been reported.10,15 The WAT-1 scores incorporate many of the reported symptoms of dexmedetomidine, however, while WAT-1 score can be a useful screening technique it cannot be the only screening tool for withdrawal from dexmedetomidine, as it does not capture all reported symptoms. Rebound hypertension and tachycardia are well-described effects of discontinuation of alpha-2 agonist, rarely requiring treatment and are not linked to withdrawal episodes ${ }^{8}$ and hence was not a focus of this study.

Our study also aimed to quantify risk factors for withdrawal with the aim of identifying patients who require a more careful wean of dexmedetomidine. As expected total cumulative dose of dexmedetomidine was a risk factor for withdrawal. This is an expected finding as relationship of dose to subsequent withdrawal is well described with other analgesics and sedatives. 'We focused specifically on total cumulative dose of dexmedetomidine prior to starting the wean as this parameter is clinically useful and can be applied by clinicians to identify which patients are at risk for withdrawal. The total cumulative dose prior to starting the wean was also not influenced by the occurrence of withdrawal symptoms. This remained a significant risk factor both in univariate and multiple logistic regressions. A cumulative dose of $107 \mathrm{mcg} / \mathrm{kg}$ prior to initiating the wean was more likely to be associated with withdrawal. In practical terms this equates to a dexmedetomidine infusion running at 1 microgram $/ \mathrm{kg} /$ hour over 4 days. This may be a possible indicator which could be used to identify which patients may require more careful weaning of dexmedetomidine in design of future studies.

We choose to include all patients who weaned from dexmedetomidine in this study including those that had weans of their other analgesics and sedatives concurrently. The reason for this is that this reflects the clinical reality of practice in critical care where drugs are concurrently weaned to progress patients through their critical illness. We acknowledge that the overlap in withdrawal symptoms with opioid and benzodiazepine is an important confounding variable however the study reflects the pragmatic practice in critical care and the challenges that clinicians face in diagnosing withdrawal when multiple agents are involved.

One other factor to take in account when weaning from dexmedetomidine is the duration of opioid use prior to initiating a dexmedetomidine wean. The longer the duration of use of opioids the more likely there was to have withdrawal in our cohort. This remained significant in univariate and multiple logistic regressions. The fact that some of the weaning courses were done with concomitant wean of opioids makes the interpretation of this risk factor more complicated. Opioids exposure could also be a useful risk factor to consider in the design of future studies testing weaning algorithms for dexmedetomidine.

While we used a number of methods to identify potential withdrawal episodes and exclude other explanations to the reported clinical change, a limitation of this retrospective study is being able to accurately attribute the observed symptoms to that of withdrawal and control for other potential contributing variables. Further, the WAT-1 withdrawal score is not validated for paediatric critical care patients receiving dexmedetomidine. The use of two reviewers helped to determine if there was any other clinical explanation for the presence of withdrawal symptoms and made the detection of withdrawal more accurate. In comparison, previous studies by Whalen etal ${ }^{10}$ and Carney etal ${ }^{4}$ reported withdrawal symptoms occurring at discontinuation of dexmedetomidine using only withdrawal scores and hence our method provided a more robust screening for withdrawal.

Clonidine has been used to prevent opioid withdrawal $^{20,21}$ and has been described as a possible transition from long-term dexmedetomidine infusion ${ }^{18}$ based on it being an oral alpha- 2 agonist. While this is plausible from a pharmacological basis, the difference 
Figure 2. Receiver operator curve curve for cumulative dose of dexmedetomidine before weaning.

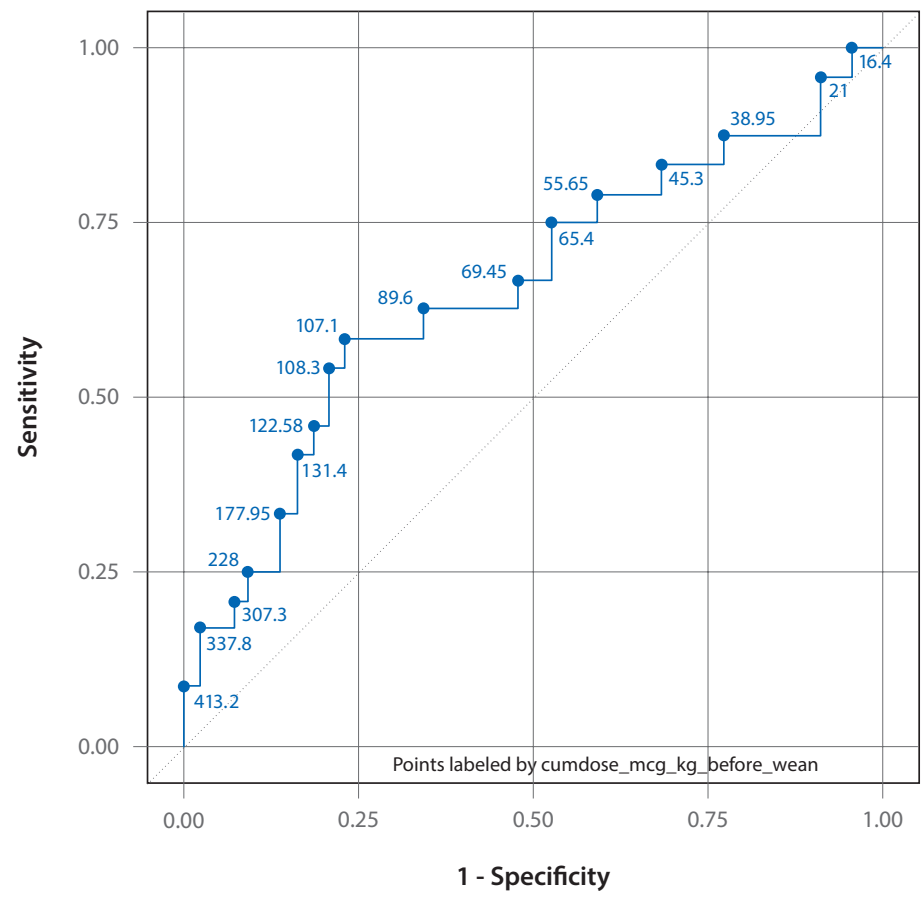

in potency between clonidine and dexmedetomidine leads to questions about efficacy and what doses would be required to achieve this goal and if this would actually provide any benefit. Despite the broad use of clonidine in our study cohort (54\% of the courses) we could not demonstrate a particular benefit in reducing the frequency of withdrawal symptoms.

\section{Conclusions}

Our study demonstrates that withdrawal syndrome does occur after prolonged infusion of dexmedetomidine. Withdrawal is possibly more likely to occur after a cumulative dose of dexmedetomidine of $107 \mathrm{mcg} / \mathrm{kg}$ (which equates to 4 days of use at a dose of $1 \mathrm{mcg} / \mathrm{kg} /$ $\mathrm{hr})$. Onset of symptoms is more likely towards the latter part of the wean or after discontinuation of the infusion. In our study, all patients were exposed to opioids and most of them had concomitant weans of dexmedetomidine and opioids; hence duration of opioids exposure may affect the occurrence of dexmedetomidine withdrawal. More work is required to examine this relationship. Future studies should focus on testing standardised weaning algorithms prospectively, and clarifying the role of clonidine.

\section{ARTICLE INFORMATION}

Affiliations Department of Critical Care Medicine (ASH, WS, PCL, CRB), The Hospital for Sick Children, Toronto, Canada; Department of Pharmacy (WS), The Hospital for Sick Children,
Toronto, Canada; Department of Diagnostic Imaging and Neuroscience \& Mental Health Program (CMU), The Hospital for Sick Children Research Institute, Toronto, Canada; Department of Clinical research services (DS), The Hospital for Sick Children, Toronto, Canada; and University of Toronto (DRB, PCL, WS), Toronto, Ontario, Canada

Correspondence Dr. A. Haenecour; astrid.haenecour@gmail.com

Disclosure The authors declare no conflicts or financial interest in any product or service mentioned in the manuscript, including grants, equipment, medications, employment, gifts, and honoraria. The authors had full access to all the data in the study and take responsibility for the integrity of the data and the accuracy of the data analysis.

Copyright Published by the Pediatric Pharmacy Advocacy Group. All rights reserved.

For permissions, email: matthew.helms@ppag.org

\section{REFERENCES}

1. Ista E, van Dijk M, Gamel C, et al. Withdrawal symptoms in children after long-term administration of sedatives and/ or analgesics: a literature review. "Assessment remains troublesome". Intensive Care Med. 2007;33(8):13961406.

2. Mason KP, Lerman J. Review article: Dexmedetomidine in children: current knowledge and future applications. Anesth Analg. 2011;113(5):1129-1142. 
3. Tobias JD. Dexmedetomidine: applications in pediatric critical care and pediatric anesthesiology. Pediat Crit Care Med. 2007;8(2):115-131.

4. Carney L, Kendrick J, Carr R. Safety and Effectiveness of Dexmedetomidine in the Pediatric Intensive Care Unit (SAD-PICU). Can J Hosp Pharm. 2013;66(1):21-27.

5. Potts AL, Anderson BJ, Warman GR, et al. Dexmedetomidine pharmacokinetics in pediatric intensive care--a pooled analysis. Paediatr Anaesth. 2009;19(11):1119-1129.

6. Summary Basis of Decision (SBD) PRECEDEX. Health Canada, Hospira Healthcare Corporation; 2010. https:// hpr-rps.hres.ca/reg-content/summary-basis-decisiondetailOne.php?linkID=SBD00112

7. Burbano $\mathrm{NH}$, Otero AV, Berry DE, et al. Discontinuation of prolonged infusions of dexmedetomidine in critically ill children with heart disease. Intensive care Med. 2012;38(2):300-307.

8. Gupta P, Whiteside W, Sabati A, et al. Safety and efficacy of prolonged dexmedetomidine use in critically ill children with heart disease. Pediat Crit Care Med. 2012;13(6):660-666.

9. Walker J, Maccallum M, Fischer C, et al. Sedation using dexmedetomidine in pediatric burn patients. J Burn Care Res. 2006; 27(2):206-210.

10. Whalen LD, Di Gennaro JL, Irby GA, et al. Long-term dexmedetomidine use and safety profile among critically ill children and neonates. Pediat Crit Care Med. 2014;15(8):706-714.

11. Reiter PD, Pietras M, Dobyns EL. Prolonged dexmedetomidine infusions in critically ill infants and children. Indian Pediatr. 2009;46(9):767-773.

12. Weber MD, Thammasitboon S, Rosen DA. Acute discontinuation syndrome from dexmedetomidine after protracted use in a pediatric patient. Paediatr Anaesth. 2008;18(1):87-88.
13. Miller JL, Allen C, Johnson PN. Neurologic withdrawal symptoms following abrupt discontinuation of a prolonged dexmedetomidine infusion in a child. $J$ Pediatr Pharmacol Ther. 2010;15(1):38-42.

14. Darnell C, Steiner J, Szmuk P, et al. Withdrawal from multiple sedative agent therapy in an infant: is dexmedetomidine the cause or the cure? Pediat Crit Care Med. 2010;11(1):e1-3.

15. Enomoto Y, Kudo T, Saito T, et al. Prolonged use of dexmedetomidine in an infant with respiratory failure following living donor liver transplantation. Paediatr Anaesth. 2006;16(12):1285-1288.

16. Hammer GB, Philip BM, Schroeder AR, et al. Prolonged infusion of dexmedetomidine for sedation following tracheal resection. Paediatr Anaesth. 2005;15(7):616-620.

17. Bejian S, Valasek C, Nigro JJ, et al. Prolonged use of dexmedetomidine in the paediatric cardiothoracic intensive care unit. Cardiol Young. 2009;19(1):98-104.

18. Tobias JD. Dexmedetomidine: are tolerance and withdrawal going to be an issue with long-term infusions? Pediat Crit Care Med. 2010;11(1):158-160.

19. Franck LS, Harris SK, Soetenga DJ, et al. The Withdrawal Assessment Tool-1 (WAT-1): an assessment instrument for monitoring opioid and benzodiazepine withdrawal symptoms in pediatric patients. Pediat Crit Care Med. 2008; 9(6):573-580.

20. Gowing L, Farrell MF, Ali R, et al. Alpha2-adrenergic agonists for the management of opioid withdrawal. Cochrane Database Syst Rev. 2014; 3:CD002024.

21. Honey BL, Benefield RJ, Miller JL, et al. Alpha2-receptor agonists for treatment and prevention of iatrogenic opioid abstinence syndrome in critically ill patients. Ann Pharmacother. 2009; 43(9):1506-1511. 